\title{
ON THE ENTROPY OF ISOMETRIES OF COMPACT METRIC SPACES
}

\author{
J. W. ENGLAND AND N. F. G. MARTIN ${ }^{1}$
}

It is known from spectral considerations that the entropy of an isometry $T$ of a compact metric space with respect to a finite invariant Borel measure is zero. In this note we compute the entropy of such metric automorphisms by constructing an increasing sequence of finite $\sigma$-fields $\left\{g_{n}\right\}$ such that $\mathrm{V}_{j=-\infty}^{+\infty} T^{j}\left(\mathrm{~V}_{n=1}^{\infty} \mathcal{S}_{n}\right)$ is the Borel field $B$ and such that the relative entropy of $T$ given $\mathcal{S}_{n}, h\left(T, \mathcal{G}_{n}\right)$, is zero for each $n$. It then follows from a theorem of Sinai [3] that $h(T)=0$.

We now proceed to the construction of the $\mathcal{S}_{n}$. Let $M$ denote a compact metric space, $T$ an isometry of $M$ and $m$ a $T$-invariant probability measure on the Borel sets $B$ of $M$. Given any sphere $S$ in $M$ with center $x$ there exists a sphere $S^{\prime}$ with center $x$ and radius less than that of $S$ whose boundary, $\partial S^{\prime}$, has measure zero. For if the boundaries of all spheres contained in $S$ and centered at $x$ had positive measure then the measure of $M$ could not be finite since all these boundaries are disjoint and there are a nondenumerable number of such sets. Thus for each $x \in M$ and each positive integer $n$, there exists a sphere $S(\epsilon(n, x) ; x)$ with center $x$ and radius $\epsilon(n, x)<1 / n$ such that $m(\partial S(\epsilon(n, x) ; x))=0$.

For each $n$, let $A_{n}=\left\{S\left(\epsilon\left(n, x_{j}^{n}\right) ; x_{j}^{n}\right): j=1,2, \cdots, k_{n}\right\}$ be a finite collection of the spheres described above which covers $M$, and define $\mathcal{F}_{n}$ to be the $\sigma$-field generated by the sets in $A_{n}$. Define $\mathcal{G}_{1}$ to be $\mathcal{F}_{1}$ and for $n \geqq 2, \mathcal{G}_{n}$ to be the $\sigma$-field generated by $\mathcal{G}_{n-1}$ and $\mathcal{F}_{n}$.

We now show that $G=V_{n=1}^{\infty} \mathcal{G}_{n}=B$. Let $U$ be an open set and $p \in U$. There is an $\epsilon>0$ such that $S(\epsilon, p) \subset U$. Select $n$ so large that $1 / n<\epsilon / 2$ and consider the open cover $A_{n}$. There is a $j$ such that $1 \leqq j \leqq k_{n}$ and $p \in S\left(\epsilon\left(n, x_{j}^{n}\right) ; x_{j}^{n}\right)$. Since $\epsilon\left(n, x_{j}^{n}\right)<1 / n<\epsilon / 2$ we have that $S\left(\epsilon\left(n, x_{j}^{n}\right) ; x_{j}^{n}\right)$ $C S(\epsilon, p)$. Thus for each $p \in U$ there is an open set $U_{p} \in \mathcal{G}$ which contains $p$ and is contained in $U$ and it follows that $U=U\left\{U_{p}: p \in U\right\}$. Since $M$ is second countable there is a countable number of the $U_{p}$ whose union is $U$. Thus $U \in \mathcal{G}$ and since $U$ was an arbitrary open set $B \subset$ G. Since $G$ is generated by open sets $G \subset B$ and we have that $\mathcal{S}=$ B.

THEOREM. If $M$ is a compact metric space, $T$ an isometry of $M$, and $m$ a $T$-invariant probability measure on the Borel sets $B$ of $M$ then the $m$-entropy of $T$ is zero.

Received by the editors March 23, 1967.

${ }^{1}$ Research supported in part by ARO Grant 662. 
Proof. Let $\mathcal{G}_{n}$ and $\mathcal{F}_{n}$ be the $\sigma$-fields constructed above. From a theorem of Sinai [3], (see also Jacobs [2]), we have that $\lim _{n} h\left(T, \varrho_{n}\right.$ ) $=h(T)$. We will show that for each integer $n, \mathcal{S}_{n} \subset V_{j=1}^{\infty} T^{-j} \mathcal{G}_{n}$ a.e. from which it follows that $h\left(T, g_{n}\right)=0$ and hence $h(T)=0$.

Since $\mathcal{G}_{n}=\bigvee_{j=1}^{n} \mathcal{F}_{j}$ is the same $\sigma$-field as the $\sigma$-field generated by the $A_{j}$ 's for $j=1,2, \cdots, n$ it is enough to show that $S\left(\epsilon\left(q ; x_{p}^{q}\right) ; x_{p}^{q}\right)$ $\in \mathrm{V}_{j=1}^{\infty} T^{-j} \mathcal{G}_{n}$ a.e. for $q=1,2, \cdots, n$ and $p=1,2, \cdots, k_{q}$.

Let $q$ and $p$ be given with $1 \leqq q \leqq n$ and $1 \leqq p \leqq k_{q}$. Denote $x_{p}^{q}$ by $y$ and $\epsilon\left(q, x_{p}^{q}\right)$ by $\epsilon$ and consider the sphere $S(\epsilon ; y)$. Since the closure of $U_{j=-\infty}^{+\infty} T^{j}(y)$ is a minimal set there exists a sequence $\left\{t_{r}\right\}$ of positive in tegers such that $t_{r} \rightarrow+\infty$ and $\rho\left(T^{-t_{r}}(y), y\right) \downarrow 0$. Since $T$ is an isometry and sends spheres into spheres of the same radius, we have

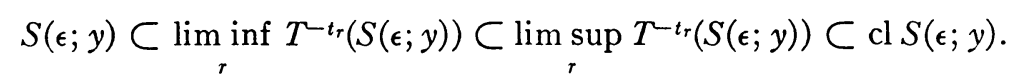

Since $m(\partial S(\epsilon ; y))=0$ it follows that $S(\epsilon ; y)=\lim \inf _{r} T^{-t_{r}}(S(\epsilon ; y))$ a.e. and hence that $S(\epsilon ; y) \in \bigvee_{j=1}^{\infty} T^{-j} \mathcal{G}_{n}$ a.e.

Corollary. If $M$ is a compact metric space and $(M, T)$ is an equicontinuous dynamical system, i.e. the family $\left\{T^{n}\right\}$ is equicontinuous then the m-entropy of $T$ is zero for every $T$-invariant Borel probability measure $m$.

Proof. If $\rho$ is a given metric on $M$, then $\rho^{\prime}(x, y)=\sup \left\{\rho\left(T^{n} x, T^{n} y\right)\right.$ : $-\infty<n<+\infty\}$ is an equivalent metric on $M$ and the equicontinuity of $\left\{T^{n}\right\}$ implies that $T$ is an isometry with respect to $\rho^{\prime}$.

The Theorem and Corollary were suggested by Examples 1 and 1 (a) of [1].

\section{BIBLIOGRAPHY}

1. Roy L. Adler, A. G. Konheim and M. H. McAndrew, Topological entropy, Trans. Amer. Math. Soc. 114 (1965), 309-319.

2. Konrad Jacobs, Lecture notes on ergodic theory, Vol. II, Aarhus Universitet, Aarhus, Denmark, 1962/1963.

3. Ja. G. Sinai, Flows with finite entropy, Dokl. Akad. Nauk SSSR 125 (1959), 1200-1202. (Russian)

UNIVERSITY OF VIRGINIA 